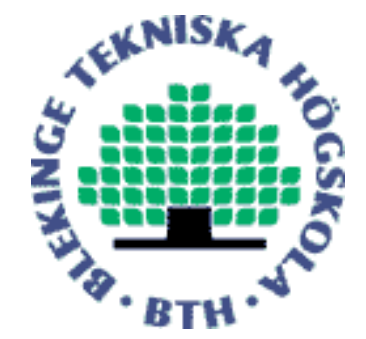

Copyright (C) 2014 IEEE.

Citation for the published paper:

An Organic View of Prototyping in Information System Development

Guohua Bai

International Conference on Computational Science and Engineering

2014 ChengDu

This material is posted here with permission of the IEEE. Such permission of the IEEE does not in any way imply IEEE endorsement of any of BTH's products or services Internal or personal use of this material is permitted. However, permission to reprint/republish this material for advertising or promotional purposes or for creating new collective works for resale or redistribution must be obtained from the IEEE by sending a blank email message to pubs-permissions@iee.org.

By choosing to view this document, you agree to all provisions of the copyright laws protecting it. 


\title{
An Organic View of Prototyping in Information System Development
}

\author{
Guohua Bai \\ Dept. Creative Digital Technology \\ Blekinge Institute of Technology \\ Sweden \\ Guohua.Bai@bth.se
}

\begin{abstract}
This paper presents an organic view of prototyping for managing dynamic factors involved in evolutionary design of information systems (IS). Those dynamic factors can be caused by, for example, continuing suggestions from users, changes in the technologies, and users-designers learning related stepwise progresses. Expanding the evolutionary prototyping to 'start small and grow', the organic view of prototyping proposes two prerequisites to do so, namely 1) a sustainable and adaptive 'embryo' - an organic structure of the future system, and 2) an embedded learning and feedback management that the actors of the system (users, designers, decision makers, administrators) can communicate with each other. An example of eHealth system design demonstrates how the prerequisites can be implemented.
\end{abstract}

Keywords-evolutionary prototyping; organic structure; embedded feedback learning, information system development

\section{INTRODUCTION}

It is a known challenge for designers of information system (IS) how to adapt the dynamic factors and wrap the diversities in developing system successively. Those dynamic factors can be caused by, for example, continuing suggestions from users, changes in the applied technologies, and users-designers learning related stepwise progresses. There is also a problem of interoperability among applications that are developed by different groups of designers. Very often there are several organizations providing similar or related services to the same group of users, such as healthcare providers and consumers. These various services and applications in different organizations can lead to the difficulty of interoperability if the service applications are not structured. In healthcare area for example, hospitals care, municipal home healthcare, and other services organizations are providing various services to senior citizens home, but using their stand-alone systems and not able to cooperate with each other even this cooperation is very needed for the seniors at home. To deal with the dynamic challenges and interoperability problem, it is very important for designers to find a strategy or methodology for the design work in the very beginning. In this paper, an organic view of prototyping is proposed to designers to deal with the above challenge. First part of the paper describes a proposed model or process in general of the design based on an organic development view. Then in the second and the third part, the two prerequisites for conducting this organic view of prototyping are discussed respectively in the context of eHealth system design. An example of design for eHealth is used to validate the applicability of this suggested approach.

\section{THE ORGANIC VIEW OF PROTOTYPING}

Prototyping as a way of design originated from industrial production, such as cars manufacturing where the goal of prototyping is to make a first type for testing before its mass production. Prototyping in this context is a well-defined prephase to the production process. When the prototype can exhibit all the essential features of the final product the prototyping process is ended, and mass-production then starts.

IS development in social and organizational contexts differs from this kind of industrial production in several ways. Firstly, the design of IS as a working tool is often part of the overall design of social and organizational work. Design of work in social and organizational contexts is much more complex and dynamic than the design of an industrial product. Such complexities and dynamics preclude the making of a prototype: a prototype can never exhibit all the essential features comprising these complexities and dynamics. Secondly, a prototype is no longer a 'first type' because the IS development process in a social and organizational design context in most cases leads to one single product, a product which is only suitable for use in this specific context [9].

We therefore need to expand on the notion of prototyping with regard to the development of IS. Floyd [9] pointed out that by using a prototype in IS development, we are primarily interested in a process rather than in a product. What we are looking for, then, are processes which lead to an early practical demonstration of relevant parts of the desired software on a computer, and which are to be combined with other processes in systems development with a view to improving the quality of the whole social and organizational system.

Another important aspect of prototyping in IS development described by Floyd is that of learning. "A prototype should always be considered as a learning vehicle providing more precise ideas about what the target system should be like".

According to Floyd [9], there are basically three kinds of purposes for prototyping in the IS development

- prototyping for exploration (exploratory prototyping), where the emphasis is on clarifying 
requirements and desirable features of the target system;

- prototyping for experimentation (experimental prototyping), where the emphasis is on determining the adequacy of the proposed solution before investing in large-scale implementation of the target system;

- Prototyping for evolution (evolutionary prototyping), where the emphasis is on adapting the system gradually to changing requirements, which cannot reliably be determined in a single early phase.

The one of evolutionary prototyping is, on the one hand, the most powerful use in information system design within organizational and social context, and on the other hand, most remote from the original meaning of 'prototyping'. The evolutionary prototyping works as an iterative feedback cycles of (re-)design, (re-)implementation, (re-)evaluation in order to accommodate continuous and unpredictable changes. Thus Floyd [9] described IS development process as such:

After an overall business analysis ('what do we want to change and why?' ), analyze the working situation and develop a model of it in terms familiar to the user; then try to elaborate the requirements specification and the design for a potential software system using the basic elements of the application field model; based on this system model, construct a prototype and let it be evaluated by the users, repeat this cycle until the necessary revisions at the end of a cycle indicate a near stable set of requirements; then build the actual target system incrementally, using the last prototype as part of its specification. (Floyd, 1984, p.15)

This paper expands this evolutionary prototyping to an organic view by embedding two fundamental prerequisites of any living system, namely 1) a sustainable and adaptive 'embryo' - an organic structure of the future system and 2) an embedded learning and feedback management that the actors of the system (users, designers, decision makers, administrators) can communicate with each other and with the environment (fig. 1). The design process described in fig. 1 is rather similar with the description in the above by Floyd and thus the focus of the paper will be on the discussion of the two prerequisites.

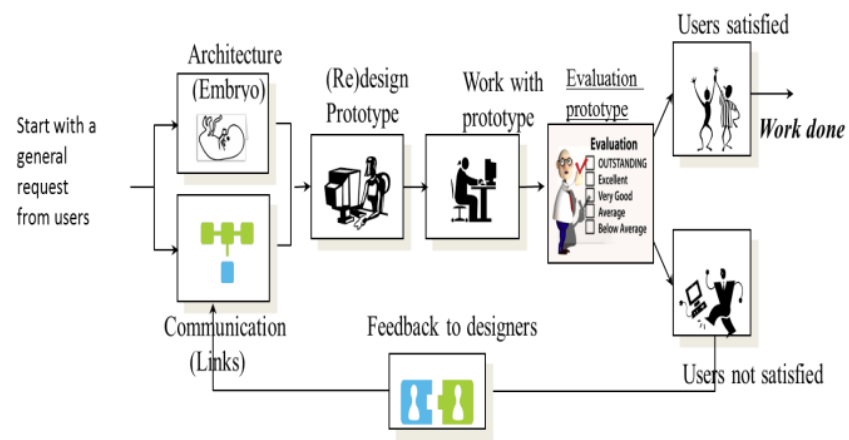

Figure 1. An organic view of evolutionary prototyping
Based upon the above two prerequisites as a start point, the evolutionary prototyping is viewed as a process of the 'embryo' growing to 'mature' [1]. The sustainable, expandable and adaptive system structure is the platform where 'growing' can be achieved. It can be regarded as a skeleton of an organic system. In practice, the effort to find this skeleton is a difficult task for the designers and it could lead to each designer to propose own structure with claiming to be the most appropriate for own specific domain. Nevertheless, the organic view of a development requires that great effort first should be made to the selection of a sustainable, expandable and adaptive system structure that is most suitable for the purpose at hand, instead of hastily engaged into the diversified contents or functions. To find this skeleton is perhaps most difficult in services such as eHealth, because the work in those fields is very poorly structured which at the same time being very dynamic. Of even great disadvantage is that the social sciences, which relate to the service activities, usually do not provide a tangible and programmable structure for service information system development. In the next section of this paper a model from the activity theory [6] is proposed as an example for such a tangible and yet expandable structure.

Another prerequisite of the organic view of prototyping is the embedded learning and communication or on-line feedback within the designed system. The On-line feedback within the designed system can provide not only the 'nutrition' for the development of the 'embryo', but also a vision, encouragement, and feeling to users, designers and other actors that 'we are all in the same boat and should work together.' It creates a sense of partnership where users' interests are taken seriously by the designers. In the followings, the two prerequisites are to be discussed in the context of eHealth ${ }^{1}$ services information system design.

\section{THE EMBRYONIC ARCHITECTURE}

The first prerequisite of the organic view of prototyping is to start with a sustainable and adaptive 'embryo' - an organic structure of the future system, a tangible model, which is a working model that affords some use, and is at the same time flexible enough to be immediately manipulated to some extent.

This paper will adopt the model (fig. 2) from the Activity Theory [6] as an example of a sustainable, expandable and adaptive structure. For many other applications of the theory readers may find for example in Software Engineering [10],

\footnotetext{
${ }^{1}$ eHealth is the use of information and communication technologies (ICT) for health. Examples include treating patients, conducting research, educating the health workforce, tracking diseases and monitoring public health (WHO, 2014).

eHealth refers to tools and services using information and communication technologies (ICTs) that can improve prevention, diagnosis, treatment, monitoring and management. It includes information and data sharing between patients and health service providers, hospitals, health professionals and health information networks; electronic health records; telemedicine services; portable patient-monitoring devices, operating room scheduling software, robotized surgery and blue-sky research on the virtual physiological human (European Commission, 2014).
} 
human-computer Interaction [13], [12], and system development [2].

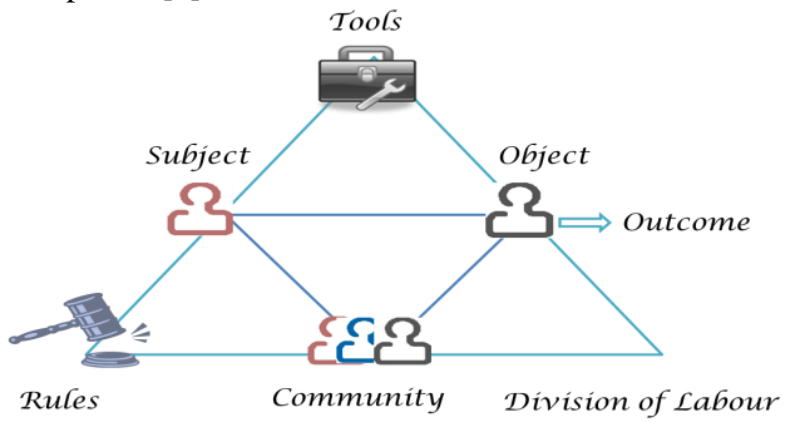

Figure 2. Activity theory model (Engeström 1978) as an embryonic structure

The model in fig. 2 has a very descriptive power for understanding how an outcome (products, services etc.) can be produced by an activity system. An activity system according to the model embodies six necessary components interrelated as an inseparable whole of any activity. The six components will be interpreted and specified as six metadatabases $^{2}$ in the followings within the context of information system design for eHealth service.

- Subject - containing service providers' databases that include information related to doctors, nurses, relatives, home services, dietitian etc.

- Object - containing service consumers' databases that include information about the consumers contact information, medical journal, diet foods, motions habits etc.

- Tools -a toolbox that includes all kinds of applications/tools for helping the healthcare, such as appointment booking, alarms, monitoring applications, diagnosing and analyzing tools, communication tools.

- Community - containing databases that link all related actors such as consumers associations, consumers advises community, other related services community, and personal contacts community.

- Rules - containing databases that include all legal, cultural and social regulations/norms in healthcare. This can also be agreements and guarantee about privacy, security, economical and service qualities.

- Labor division - containing databases that specify who (Healthcare providers) do what (to the Healthcare consumers). This specification is to guarantee that all kinds of needs of the patients have been completely and seamlessly supplied by the healthcare communities.

\footnotetext{
2 Metadatabase is a database model $\cdots$ to include such data and knowledge representation as information models (e.g., relations, entities-relationships, and objects), application logic (e.g., production rules), and analytic models. (Wikipedia,2014)
}

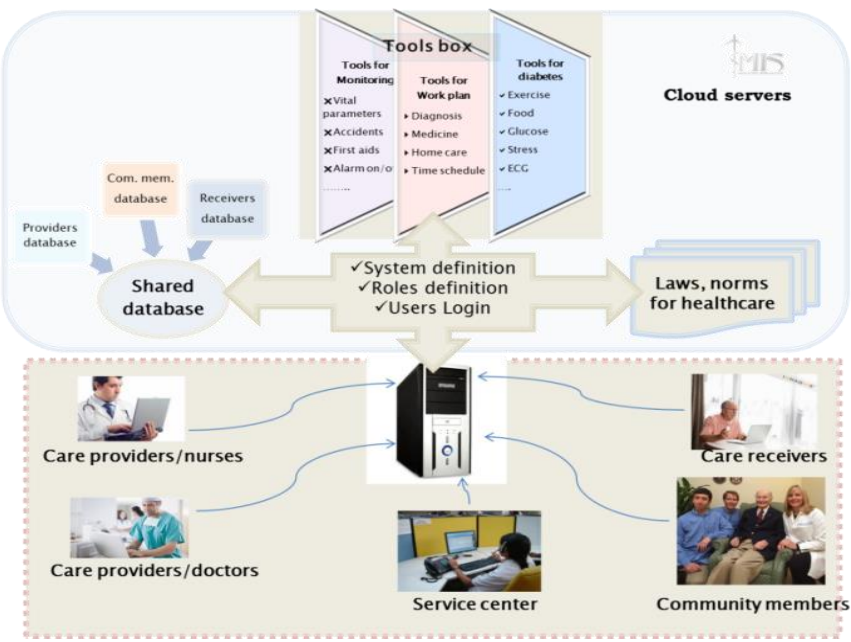

Figure 3. eHealth system based on the embryonic structure

Based on the above specified meta-databases, a project sponsored by Sweden's Innovation Agency (Vinnova) called IMIS (Integrated Mobile Information System for Healthcare) is being conducted to apply the activity theory model as an organic structure of the future system. Through a hybrid cloud platform the project is to implement the different parts of the systems by different kinds of clouds (such as public, private, and community clouds) based upon the data properties, such as security, privacy, accessibility, ownership, and reliability. A survey of the applicability of the hybrid cloud platform for design of eHealth service can be found in another paper [15]. Some concrete results will be successively published after the project is concluded.

\section{EMBEDDED LEARNING AND ON-LINE FEEDBACK WITHIN THE DESIGNED SYSTEM}

An embedded learning and on-line feedback within the designed system is the second prerequisite of the organic view of prototyping. In principle, prototyping promotes communication among actors of the team: designers, users, decision makers, and other social actors. As the design progresses, the team members communicate and thereby learn from each other. However, this manner of mutual learning through offline communication can meet some problems.

One problem is the difficulty for the users and designers to describe or to make clear statements about their work routines and their experience which is often called tacit knowledge [3]. We usually have difficulties to describe about what we do and the way we do it except for when we are in the middle of doing it and describing it within actual context. Both users and designers carry out much work based on this kind of tacit and situated knowledge and in many cases they need to represent this kind of knowledge to each other in order to co-design the IS. Perhaps the most effective way to obtain such knowledge and skill is by 'doing together'. Methods as observation, interview, ethnography study etc. may be used for this learning, however, 'situated communication' that allows them together directly seeing 
and doing the situated topics and objects can be great help for the mutual learning. The proposed online feedback in this paper provides such a situated communication and can contribute to learning and tacit knowing sharing during the evolutionary prototyping. The organic view of prototyping suggests setting up situations from the very beginning in which it is possible for the design actors to gain hands-on experience with the system, instead of developing a full scale system and accumulating many problems and difficulties for both users and designers.

To practically implement this situated communication in the evolutionary design, the paper introduces a simple mechanism for such a feedback when few users are involved in the design process (fig. 4). The workable prototype is placed on the top of screens and the embedded feedback is designed within each screen and place in the bottom where the users, designers and other actors can write down their feedback to each other about the system (prototype). All feedback messages will be automatically associated with an identifier about the targeted objects (such as buttons, menus, pages, login users etc.).

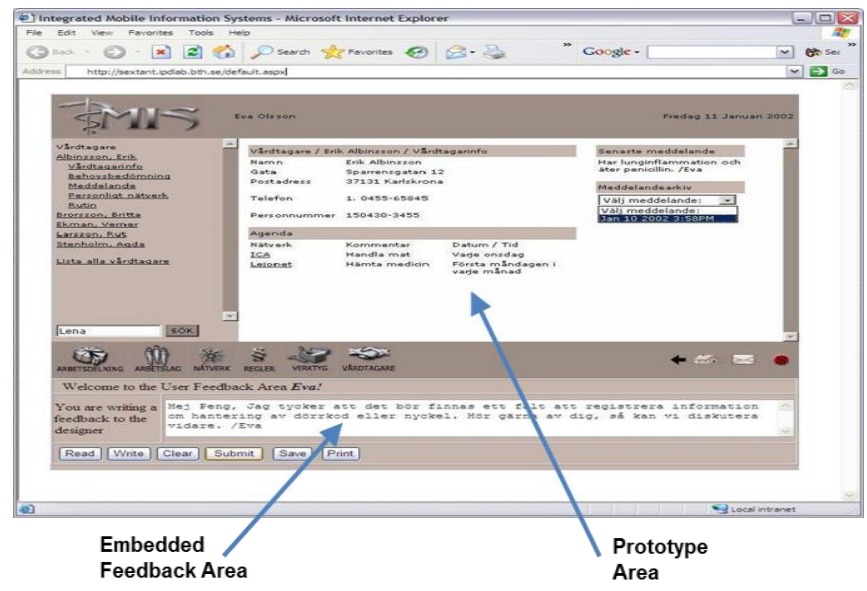

Figure 4. A simple embedded feedback and learning (from IMIS prototype)

When the designed system is becoming more complex and multiple actors are involved in, the embedded feedback has to be structured in order to keep tracking and subject oriented responding. There could be many ways to structure the feedback, and in this paper feedback will be categorized according to a category by the activity theory. According to this theory tools are important artefacts in mediating any activity, such as IS design activity that is conducted by the subject (team of design) to achieve an outcome (users satisfied IS). Engeström [7], [8] developed a hierarchy or multiple levels of mediating artefacts, and some researchers [10], [4] also interpreted and applied the hierarchy of mediating artefacts. The interpretation and how to apply this category is still open for discussion. The paper is to offer a suggestion with some examples of how the different levels of artefacts can be used in categorizing the feedback of the design based on the suggested categories (in fig. 5 the word 'tool' is used as a synonym of 'mediating artefacts' for consistency with the theoretical model in fig. 2).

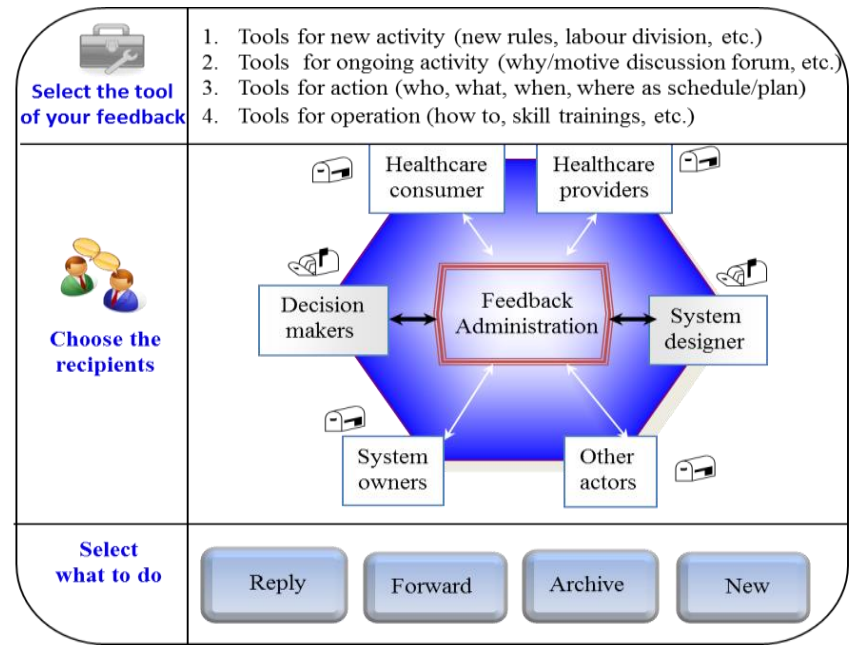

Figure 5. Advanced and multiple levels of tools for categorizing feedback

Coupling feedback directly into the designed system, misunderstandings and confusion between users, designers and other actors can be greatly reduced, because all concepts and objects mentioned in a feedback message are always related to a concrete context presented in the related interface. It should be mentioned also that the response to feedback should be in a timely fashion, especially from the designers to the users. It is recommended that the revision based on the users' feedback should be done no longer than two weeks. Some small bugs should be fixed within one or two days. The point is to make the users feel that they are making progress in the system development.

This embedded feedback and communication link provides a dynamic input to the development of the evolutionary prototype. It is a channel to enable learning and developing the 'embryo' to the future system.

\section{CONCLUSION}

How to manage dynamic factors involved in evolutionary prototyping is vital for IS systems' acceptance and usability [5]. A phrase commonly used to describe evolutionary design is to 'start small and grow'. Unfortunately, few authors provide any idea of how to accomplish this [4]. Major questions are raised, such as 'where to start?' and then 'how to let the system grow?' This paper proposed an organic view of the evolutionary prototyping by introducing two vital prerequisites based on any living system, namely 1) a sustainable and adaptive 'embryo' - an organic structure of the future system, and 2) an embedded learning and feedback. By starting with an embryonic structure of the future system and growing by feedback learning, the evolutionary prototyping can be seen as an organic process. The paper demonstrated how the approach can be implemented by an example of a project IMIS for eHealth system. 


\section{REFERENCES}

[1] G. Bai, "Embryonic approach to the development of information systems," J. Strateg. Inf. Syst., vol. 6, no. 4, pp. 299-311, 1997.

[2] G. Bai and L.-Å. Lindberg, "Dialectical approach to systems development," Syst. Res. Behav. Sci., vol. 15, no. 1, pp. 47-54, 1998.

[3] A. Clement and P. Van den Besselaar, "A retrospective look at PD projects," Commun. ACM, vol. 36, no. 6, pp. 29-37, 1993.

[4] P. Collins, S. Shukla, and D. Redmiles, "Activity theory and system design: A view from the trenches," Comput. Support. Coop. Work CSCW, vol. 11, no. 1-2, pp. 55-80, 2002.

[5] A. Dix, J. Finlay, G. Abowd, and R. Beale, Human-computer Interaction. Upper Saddle River, NJ, USA: Prentice-Hall, Inc., 1997.

[6] Y. Engeström, "Learning by expanding: An activity-theoretical approach to developmental research," 1987.

[7] Y. Engeström, Learning, working and imagining: Twelve studies in activity theory. Orienta-konsultit, 1990.

[8] Y. Engeström, "Enriching the theory of expansive learning: Lessons from journeys toward coconfiguration," Mind Cult. Act., vol. 14, no. 1-2, pp. 23-39, 2007.
[9] C. Floyd, "A systematic look at prototyping," in Approaches to prototyping, Springer, 1984, pp. 1-18.

[10] G. Georg, "Activity Theory and its Applications in Software Engineering and Technology," Colo. State Univ. Tech. Rep. CS-11101, 2011.

[11] S. Harker, "User Participation in Prototyping," Commun ACM, vol. 36, no. 6, p. 77-, Jun. 1993

[12] K. Kuutti, "Activity theory as a potential framework for humancomputer interaction research," Context Conscious. Act. Theory Hum.-Comput. Interact., pp. 17-44, 1996.

[13] B. A. Nardi, "Studying context: A comparison of activity theory, situated action models, and distributed cognition," Context Conscious. Act. Theory Hum.-Comput. Interact., pp. 69-102, 1996.

[14] W. Rouse and J. Valusek, "Evolutionary design of systems to support decision making," Decis. Mak. Action Models Methods, pp. 270-286, 1993.

[15] Y. Hu and G. Bai, "Building up a Virtual Community for HomeBased Chronic Diseases Healthcare," UsARE2014, pp. 40-43, 2014. 